\title{
Fast Opening Switch Approach for High-Voltage Vacuum Tube Protection Application
}

\author{
Wolfhard Merz ${ }^{1}$, and Monty Grimes ${ }^{2}$ \\ ${ }^{1}$ DESY, Hamburg, Germany, wolfhard.merz@desy.de \\ ²Behlke Power Electronics LLC, Billerica, MA, USA, monty.grimes@behlke.com
}

\begin{abstract}
The operation of high-power, high-frequency vacuum tubes requires an appropriate protection method to avoid significant damages during arcing. Fast closing switches like spark gaps, thyratrons, ignitrons and semiconductors acting as chargediverting bypass switches are the most commonly used protection method. These "crowbar" switches cause hard transient conditions for all subcomponents involved and usually result in a significant post-fault recovery period. The availability of fast high-voltage semiconductor devices, with flexible on/off control function, makes opening switch topologies possible and attractive to improve this situation. This paper describes a circuit topology to protect an Inductive Output Tube which is expected to operate within RF subsystems for accelerator applications. The topology is characterized by using a commercial available high voltage MOSFET switch with direct liquid cooling and completed with essential snubber extensions. The advantages of the opening switch approach are faster action, smaller fault energy, faster recovery, and more compact design. Initial test results of this topology are presented.
\end{abstract}

Keywords-component; opening switch, semiconductor, tube protection

\section{INTRODUCTION}

Recent applications of RF subsystems for particle accelerators are often characterized by limited space close to the accelerating modules inside the tunnel. Therefore very compact systems with modular architecture are required. The operation of RF systems based on vacuum electron devices is only possible with an appropriate protection system for the tube applied. This system must fit for fast action at low fault energy and compact mechanical design as well. The European XFEL, which is under construction at the DESY Research Center Hamburg, is a designated project for an upgrade option based on the application of continuous wave (cw) or long pulse (lp) RF subsystems in the future. The main RF power stage of such a subsystem comprises a $1.3 \mathrm{GHz}$ Inductive Output Tube (IOT) with $85 \mathrm{~kW}$ cw output power capability.

\section{MAIN ChARACTERISTIC}

\section{A. Operating Conditions}

The main requirements of the protection system are determined by the electrical operating conditions of the RF power stage. The ratings are $48 \mathrm{kV}$ dc operating voltage, $3.8 \mathrm{~A}$ dc operating current and fault energy smaller $10 \mathrm{~J}$ during arcing. The operating modes applied are $\mathrm{cw}$ and pulsed mode as well. Pulsed mode operation is characterized by $1 \mathrm{~Hz}$ repetition rate and duty factor ranging from 0.1 to 0.5 respectively.

\section{B. Preceding Closing Switch Protection}

A preceding installation for operating a prototype of the IOT within a Cryogenic Module Test Bench (CMTB) was accomplished by the application of the classic closing switch approach by means of Light Triggered Thyristors (LTT) [1]. For sufficient margin in case of arcing, additional current limiting resistors had to be applied. The simplified topology is given in Fig. 1. The protection efficiency of this previous test configuration will be compared with the new opening switch approach. The general topology operating an opening switch as a series connected device replacing the closing switch is given in Fig. 2. The design goal is to operate without any additional current limiting resistor to minimize heat loss during $\mathrm{cw}$ and long pulse operation.

Closing Switch

LTT crowbar

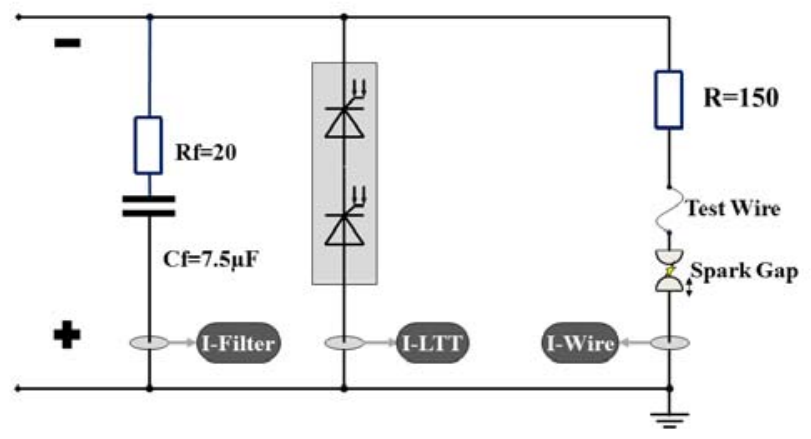

Figure 1. Preceding test installation operating a LTT crowbar

\section{Main Components for Opening Switch Protection}

Compared to high power cw RF systems usually in operation for accelerators, the moderate parameters of the IOT RF system make a compact, commercially-available opening switch approach based on a series-connected high voltage semiconductor module feasible. For proof of principle the first preference was commercial availability of the main components. 


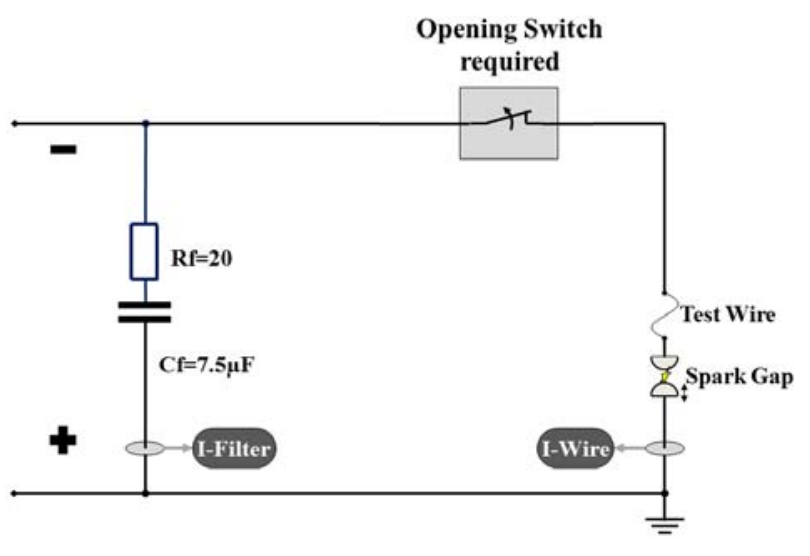

Figure 2. General topology operating an Opening Switch

\section{1) Semiconductors and Auxiliaries:}

For fast switching operation a BEHLKE High-Voltage Transistor Switch type HTS 701-10-LC2 based on MOSFET technology in combination with external control electronic is chosen. Because of the heat load of dc mode operation, a direct liquid cooled version is deemed necessary. This requires the use of some additional auxiliary components such as a pump unit with integrated electronic, active radiator, fittings, flexible tubes and Perflourinated Polyether coolant (PFPE). Main electrical data for the switch are given in Table I.

TABLE I. MAIN PARAMETERS SWITCH MOdULE

\begin{tabular}{|l|l|c|c|}
\hline \multicolumn{5}{|c|}{ HTS 701-10-LC2 (direct liquid cooling) } \\
\hline Symbol & \multicolumn{1}{|c|}{ Specification } & Value & Unit \\
\hline$V$-max & Max. Operating Voltage & 70 & $\mathrm{kV}$ DC \\
\hline$I$-pmax & Max. Turn-On Peak Current & 100 & $\mathrm{~A}$ \\
\hline$I$-l & Max. Continuous Load Current & 4.75 & $\mathrm{~A} \mathrm{DC}$ \\
\hline$t$-on & Turn-On Rise Time & 50 & $\mathrm{~ns}$ \\
\hline$t$-off & Turn-Off Rise Time & 80 & $\mathrm{~ns}$ \\
\hline$C$-c & Coupling Capacitance & 46 & $\mathrm{pF}$ \\
\hline$C$-n & Natural Capacitance & 20 & $\mathrm{pF}$ \\
\hline
\end{tabular}

The MOSFET module has been modified compared to the standard version by means of additional series diodes to protect the intrinsic body diodes from reverse current. Because of the dc current load the additional diodes need to be integrated inside the module for active liquid cooling. The main switch module is controlled by an external control unit. This feature assists in the convenient change of control-circuit parameters without the redesign of the entire switch. With the electronic unit three interface commands are established. CONTROL for usual On-Off command, /INHIBIT for fast off command related to customers fault condition and FAULT as status information in case of any internal faults of the module. A 80 kV Fast Diode Assembly (Behlke FDA 800-75) antiparallel connected to the module bypasses possible reverse current.

\section{2) Snubber Unit}

The operation of the High Voltage Transistor Switch (HTS) for short circuit conditions (tube arcing) is only possible with an additional series connected snubber unit. This unit limits the rate of short circuit current rise during arcing by means of an appropriate inductance. The HTS has to be switched off during arcing by the control electronics fast enough before reaching a critical current level that might damage the output transistors. The energy stored in the current limiting inductance has to be dissipated by a freewheeling diode-resistor combination.

\section{Test Circuit Topology}

To evaluate the switching performance of the opening switch approach a test topology according Fig. 3 was applied. The circuit comprises the switch module with bypass diode, snubber unit, an additional high voltage thyristor module to emulate short circuit conditions and some electronics controlling the switching sequence. Fault current sensing is accomplished with a Pearson monitor at low side and an appropriate level comparator to generate the /INHIBIT signal for a fast off command of the HTS.

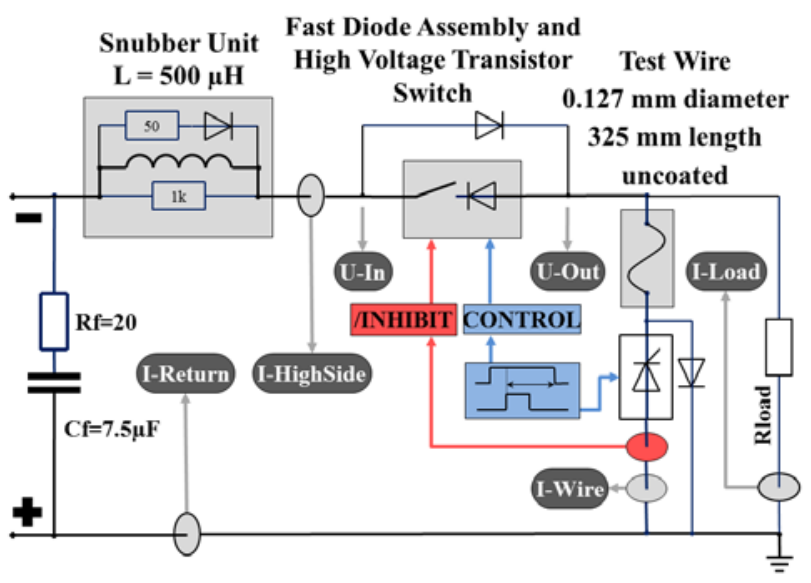

Figure 3. Test circuit topology for the wire test

\section{Test Results}

\section{A. Wire Test}

A wire test has been prepared to prove that the energy transfer during arcing is well below the $10 \mathrm{~J}$ limit allowed. The uncoated copper wire with a diameter of $0.127 \mathrm{~mm}$ and a length of $325 \mathrm{~mm}$ has been specified by the manufacturer of the tube. Closing the short circuit a fast high voltage thyristor module (Behlke HTS 800-100-SCR) was used. This module is able to withstand $80 \mathrm{kV}$ at a maximum turn-on peak current of $1 \mathrm{kA}$ for $100 \mu \mathrm{s}$, which is sufficient for the overvoltage and pulse current expected during testing. To protect the thyristor module from reverse transients an additional Fast Diode Assembly (FDA 800) was added. By means of programmable electronics an appropriate switching sequence was applied. This was desirable to get an additional off command if /INHIBIT command fails. The wire test was repeated many times at nominal voltage without any degradation of the wire. 
Current and voltage waveforms measured with this circuit topology at different operating voltages are given in Fig. 4.

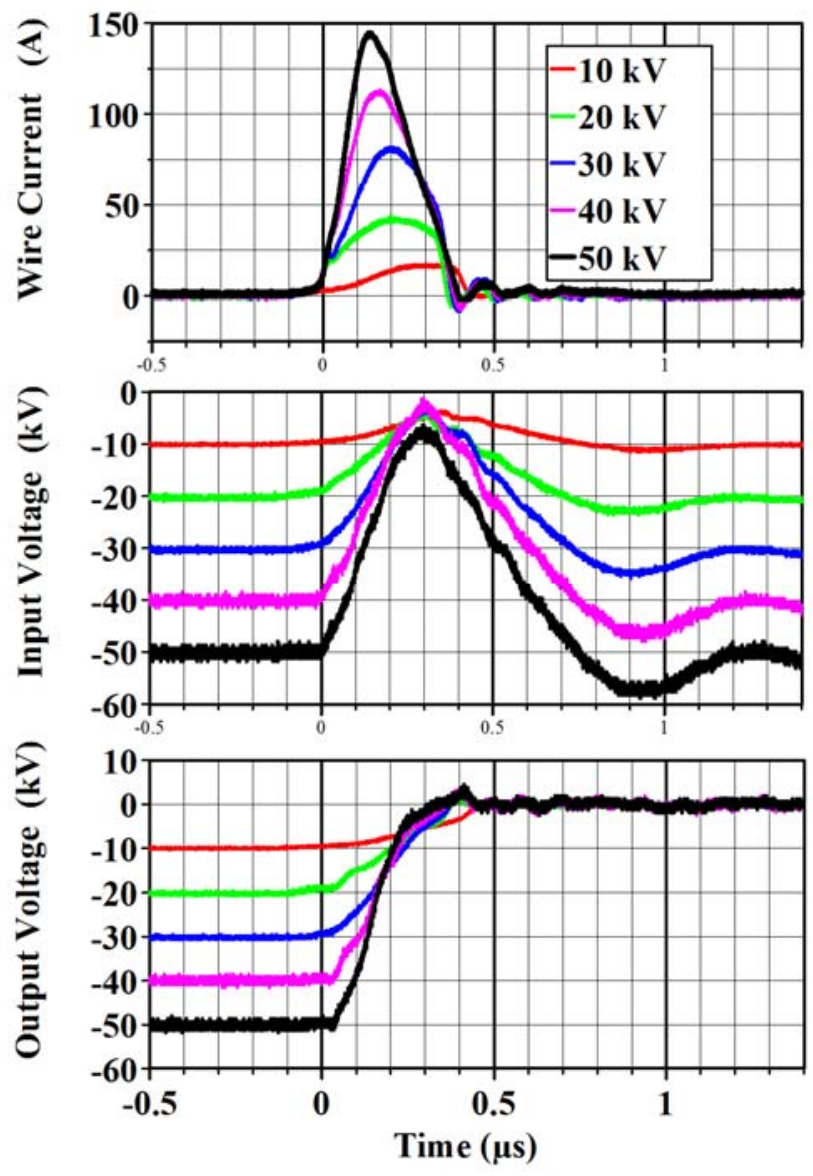

Figure 4. Wire test at different voltages

The current peak measured through the wire at nominal voltage exceeds the maximum peak current specified for the switch module. This was an initial concern for further tests. To verify the actual dynamic current load of the module a second current sensor was added at the input side of the switch. This additional measurement of the current pulse at high side gives significant deviation compared to the low side measurement for the wire. An example of the resulting voltage and current waveforms are given in Fig.5. Obviously additional charge transfer by parasitic capacitances within the test topology is responsible for this effect. Based on the measured current deviation, the calculation of the transferred charge at nominal voltage gives approximately $225 \mathrm{pF}$ for the capacitance considered. The high side measurement proves that short circuit operation fits the 100 A current rating of the switch module.

\section{B. Snubber Unit Decoupling Feature}

To prove the essential decoupling function of the snubber unit, the return current with respect to wire current was measured during short circuit operation. The waveforms are given in Fig. 6. The current rise of return current correlates the expected value of 100 A per microsecond. For proof of principle the parasitic oscillations observed are not considered within this context.

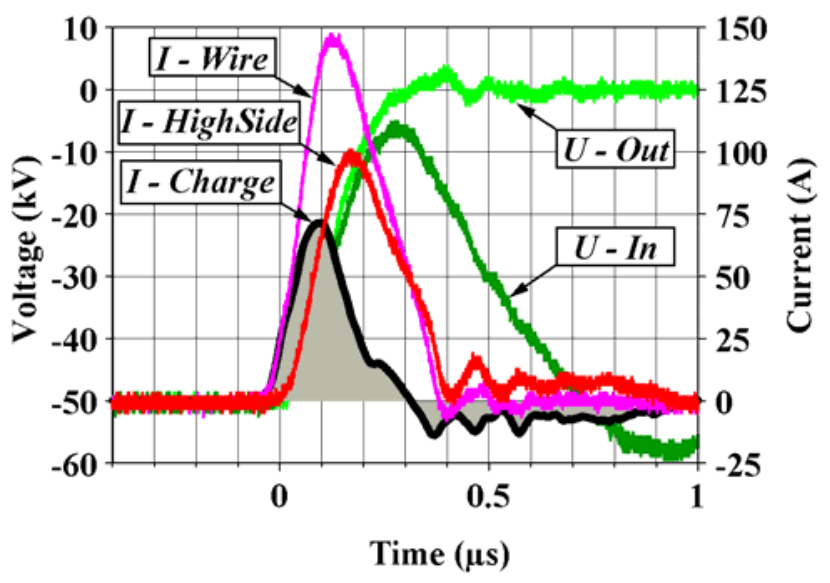

Figure 5. Additional charge transfer current during wire test calculated by the difference of measured wire current and high side current respectively

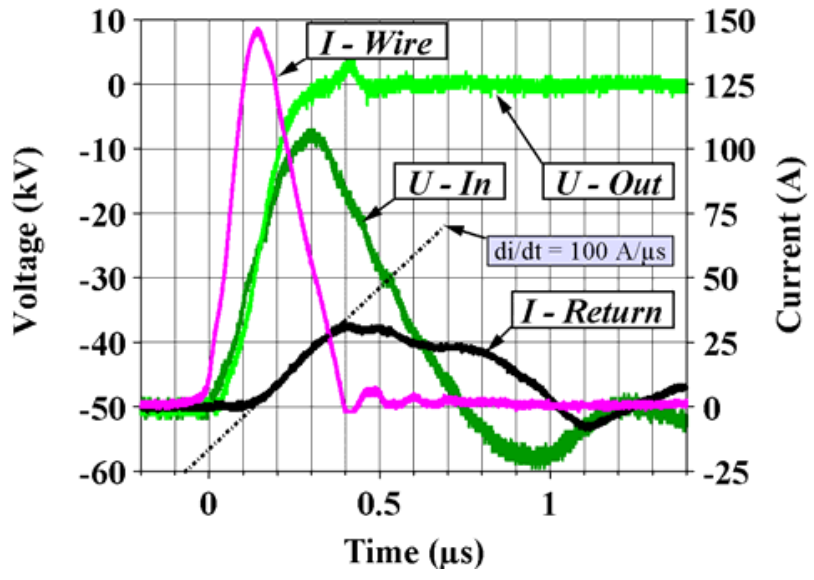

Figure 6. Contrasting the waveforms of wire current and return current during short circuit operation at nominal voltage

\section{Fault Current Charge Transfer}

To demonstrate the significant improvement achievable with the new opening switch approach compared to the preceding closing switch crowbar the charge $(Q)$ transferred through the test wire has been identified. In Fig. 7 the charge transfer through the test wire for both opening and closing switch during wire test at nominal voltage are presented. It is important to recognize that the opening switch topology operates without any series connected current limiting resistors. This is of special importance to reduce the additional heat loss during cw and long pulse operation of the tube. 


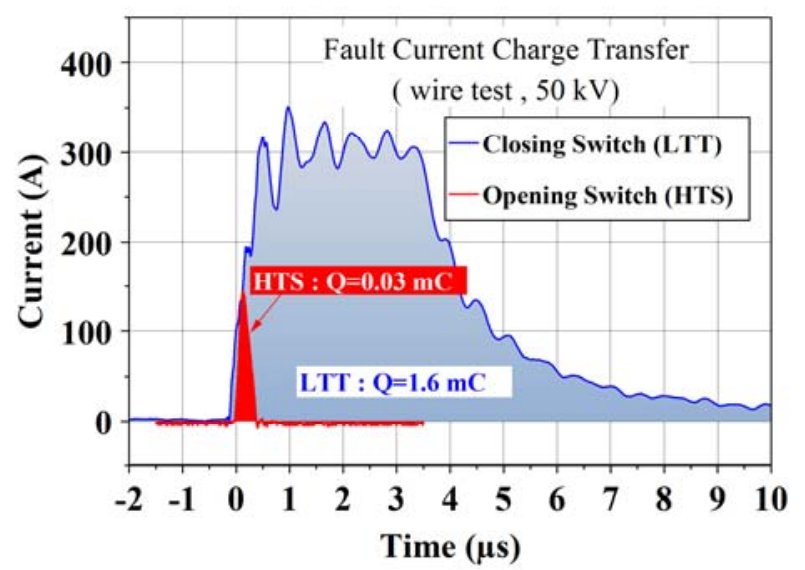

Figure 7. Charge transfer through the test wire during short circuit operation at nominal voltage

\section{Long Pulse Operation}

Due to power limitations with the dummy load long pulse operation was only possible for a preliminary demonstration by applying On-Off command to the opening switch. An example is given in Fig. 8.

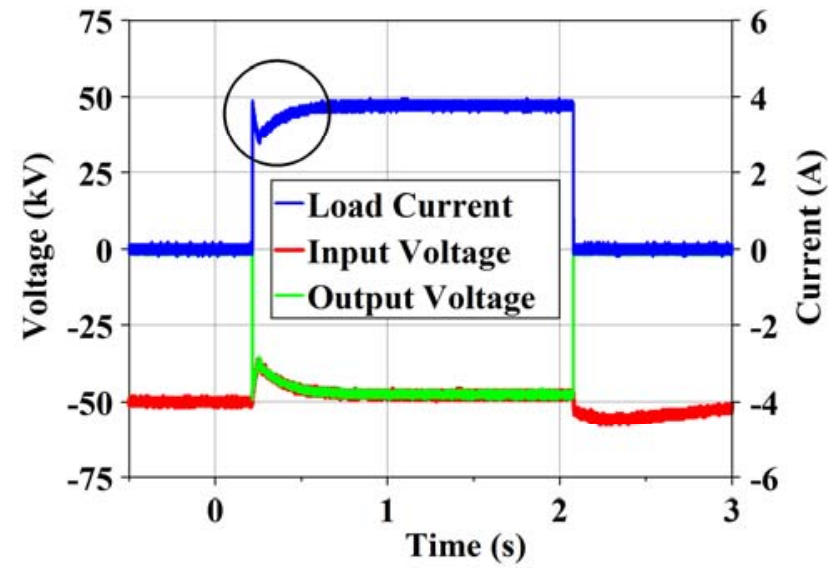

Figure 8. Long pulse operation at nominal load condition

The drop at the beginning of the pulse is caused by the inadequate power supply, which is optimized for clean dc operation only. To improve this situation an appropriate upgrade is underway. At the final design the long pulse mode will be controlled by the RF drive of the IOT. The opening switch has to withstand all resulting load conditions only and finally to protect the tube in case of arcing.

\section{Conclusion}

The initial test results of this work prove the feasibility of the opening switch approach based on compact semiconductor modules for tube protection applications. The requested maximum fault energy smaller $10 \mathrm{~J}$ can be accomplished easily. In general there is significant potential of this circuit topology to achieve even smaller fault energy levels. This work is considered as a first step taken to prove the principle.

For further investigations and development there are some complex details left to be solved:

- The very fast switching speed of the MOSFET module under high voltage conditions causes significant interaction with stray capacitances and leakage inductances of the circuit topology. An improved construction of the circuit layout has to avoid possible resonant transients intrinsically.

- For a very compact mechanical design alternate housing and cooling methods of the switch module in strong relation to the snubber unit needed have to be investigated.

- High side current sensing for fault level detection and fast /INHIBIT command is the preferred solution. Solving the contradiction of sensitivity and robustness against electromagnetic interference is a challenging task. The availability of fast current monitors with dc capability is mandatory.

- The overall reaction time of the system is $400 \mathrm{~ns}$ for now. Further improvement, if desired, is possible with a redesign of the external control unit to get faster response time for the /INHIBIT interface.

\section{ACKNOWLEDGMENT}

We want to express our gratitude to B. Albrecht, R. Gabler, S. Knoop and T. Wölflick, who are members of the DESY high voltage dc power supply group, for their technical support preparing the test setup.

\section{REFERENCES}

[1] W. Merz, J.P. Jensen ”Light Triggered Thyristor Crowbar for Klystron Protection Application”, Proceedings of the PAC 2003, pp. 749-751 